University of Wollongong

Research Online

Faculty of Engineering - Papers (Archive)

Faculty of Engineering and Information

Sciences

$1-1-2010$

\title{
The resonant tunneling through a graphene multiquantum well system
}

G.J Xu

Chinese Academy of Sciences

X.G Xu

Shanghai Institute

B.H Wu

Chinese Academy of Sciences

J.C Cao

Shanghai Institute

Chao Zhang

University of Wollongong, czhang@uow.edu.au

Follow this and additional works at: https://ro.uow.edu.au/engpapers

Part of the Engineering Commons

https://ro.uow.edu.au/engpapers/1865

\section{Recommended Citation}

Xu, G.J; Xu, X.G; Wu, B.H; Cao, J.C; and Zhang, Chao: The resonant tunneling through a graphene multiquantum well system 2010, 123718-1-123718-6.

https://ro.uow.edu.au/engpapers/1865

Research Online is the open access institutional repository for the University of Wollongong. For further information contact the UOW Library: research-pubs@uow.edu.au 


\section{AIP Apoines Physis}

\section{The resonant tunneling through a graphene multiquantum well system}

G. J. Xu, X. G. Xu, B. H. Wu, J. C. Cao, and C. Zhang

Citation: J. Appl. Phys. 107, 123718 (2010); doi: 10.1063/1.3445782

View online: http://dx.doi.org/10.1063/1.3445782

View Table of Contents: http://jap.aip.org/resource/1/JAPIAU/v107/i12

Published by the American Institute of Physics.

\section{Related Articles}

Temperature dependence of droop onset in optically pumped intrinsic InGaAs/InP heterostructures Appl. Phys. Lett. 102, 081123 (2013)

Enhancement of multisubband electron mobility in parabolic AlxGa1-xAs-GaAs double quantum well structures J. Appl. Phys. 113, 083704 (2013)

Controlling wave function localization in a multiple quantum well structure

J. Appl. Phys. 113, 054310 (2013)

The effect of InGaN underlayers on the electronic and optical properties of InGaN/GaN quantum wells Appl. Phys. Lett. 102, 041115 (2013)

The effective g-factor in In0.53Ga0.47As/In0.52AI0.48As quantum well investigated by magnetotransport measurement

J. Appl. Phys. 113, 033704 (2013)

\section{Additional information on J. Appl. Phys.}

Journal Homepage: http://jap.aip.org/

Journal Information: http://jap.aip.org/about/about_the_journal

Top downloads: http://jap.aip.org/features/most_downloaded

Information for Authors: http://jap.aip.org/authors

\section{ADVERTISEMENT}

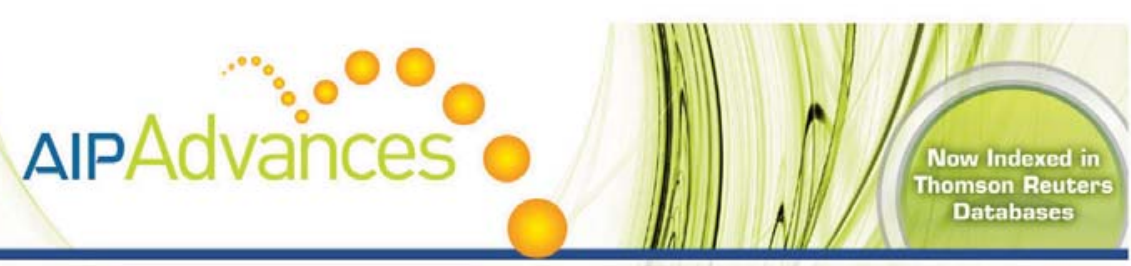

\section{Explore AIP's open access journal: •Rapid publication \\ - Article-level metrics \\ Post-publication rating and commenting}




\title{
The resonant tunneling through a graphene multiquantum well system
}

\author{
G. J. Xu, ${ }^{1}$ X. G. Xu, ${ }^{1}$ B. H. Wu, ${ }^{1}$ J. C. Cao, ${ }^{1, a)}$ and C. Zhang ${ }^{2}$ \\ ${ }^{1}$ State Key Laboratory of Functional Materials for Informatics, Shanghai Institute of Microsystem and \\ Information Technology, Chinese Academy of Sciences, 865 Changning Road, Shanghai 200050, China \\ ${ }^{2}$ School of Engineering Physics, University of Wollongong, New South Wales 2552, Australia
}

(Received 16 January 2010; accepted 10 May 2010; published online 29 June 2010)

\begin{abstract}
The transport properties of a graphene multiquantum well system are investigated numerically using transfer-matrix method. There are transmission gaps for electrons and holes in the transmission spectra at tilted incidence. In the transmission gaps, a few resonant tunneling peaks appear, defined as transmission windows, which are related with the bound states in the quantum wells. Unlike conventional semiconductor nanostructures, the location and the width of the transmission windows are sensitive not only to the quantum well width but also the incident angle. The number of the quantum wells determines the fine structure of the transmission windows. The anisotropic property is affected in the following way: the increase in well width makes the nonzero-transmission incident angle range decrease, and the interference effect is enhanced as the well number increases. Tiny oscillation of the conductance and fine structures in the middle energy range are due to the resonant tunneling induced by the multiquantum well structure. These oscillating features may be helpful in explaining the oscillatory characteristics in experiment. (C) 2010 American Institute of Physics. [doi:10.1063/1.3445782]
\end{abstract}

\section{INTRODUCTION}

The recent realization of stable monolayer graphene has attracted considerable attention on the study of its electron properties. ${ }^{1}$ This two-dimensional honey-comb structure of the graphene crystal has many unique properties which are unseen in other materials. The gapless linear dispersion close to the Dirac points and the chiral nature of the charge carriers are the origins of many exotic physical phenomena, ${ }^{1-3}$ such as Klein paradox, ${ }^{1,2,4-9}$ the odd-integer-Hall-effect, ${ }^{1,3,10,11}$ finite minimal conductivity, ${ }^{1,12}$ and specular Andreev reflection $^{13-15}$ on superconductor-graphene interface.

One merit of graphene relies on the fact that the potential profile can be modulated by applying a local gate voltage $\mathrm{e}^{16,17}$ or by adsorbing adatoms on its surface. This tunability of the band structure arose much interest to the graphene or carbonbased electronics devices such as graphene quantum dot, quantum point contact, quantum well, ${ }^{18,19}$ or superlattice, ${ }^{20-24}$ which have their prototypes in the wellstudied semiconductor nanoelectronics. In semiconductor quantum wells, it is well-known that there will be bound states localized in the potential well. When the energy of the incident electrons is aligned to the bound states, the transmission may be greatly enhanced by the resonant tunneling. When multiquantum wells are coupled to each other, the bound states between two adjacent barriers form minibands by coupling to each other. It can be expected that the same phenomenon can be observed in quantum wells made by graphene. In this study, we are interested in the resonant coherent transport through graphene nanostructures via the bound states. The charge carriers in graphene are both electrons and holes, or called positrons, owing to the chirality of graphene. ${ }^{2}$ The barriers for electrons become wells for holes

${ }^{a)}$ Electronic mail: jccao@mail.sim.ac.cn. in graphene. When the wave function is an evanescent wave in graphene barriers, a transmission gap emerges in the transmission spectra for non-normal incidence. ${ }^{25,26}$ We consider a barrier-well system to examine the property of charge carriers in graphene. In our work, transfer-matrix method mo,21,26 $^{20}$ will be explored to investigate the transport properties of a graphene multiquantum well system. The transmission probability, the anisotropic transmission, and conductance for different quantum well number and varying quantum well width will be calculated and discussed in details. The rest of our work is organized as follows: the model and theory of graphene multiquantum well will be described in Sec. II; the numerical results will be presented and discussed in Sec. III; at last, a conclusion will be derived in Sec. IV.

\section{MODEL AND FORMALISM}

Graphene is a single layer of carbon atoms densely packed in a honeycomb lattice, which consist of two interpenetrating triangular sublattices often labeled $A$ and $B$. The charge carriers in the pristine graphene can be described by massless Dirac equation close to the Dirac points K and $\mathrm{K}^{\prime}$. The linear dispersion relation $|E|=\hbar v_{F}|k|$ in the band structure is of significance in the electron properties, where $v_{F}$ $\approx 10^{6} \mathrm{~ms}^{-1}$ is the Fermi velocity and $k$ is the Fermi wavevector. The wave function can be represented by twocomponent spinor, ${ }^{1,2,13,26,27}$ which corresponds to the amplitude of the two different triangular sublattice $A$ and $B$.

We consider a graphene multiquantum well system at zero temperature and in the absence of carrier-phonon and spin-orbit interactions. The sample width is assumed to be large enough that the edge effects can be neglected. ${ }^{28}$ The massless Dirac equation can be written as 


$$
\left[v_{F}(\sigma \cdot p)+U(x)\right] \Psi=E \Psi,
$$

where the components of pseudospin $\sigma=\left(\sigma_{x}, \sigma_{y}\right)$ are Pauli matrices, $p=\left(p_{x}, p_{y}\right)$ is the in-plane momentum operator, and $U(x)$ is the one-dimensional potential along the direction of the quantum wells. Therefore, the wave function can be written in the form $\Psi=\Phi(x) e^{i k_{y} y}$, where $k_{y}=\left(E-U_{L}\right)$ / $\left(\hbar v_{F}\right) \sin \beta$ is the transversal wavevector, and $\beta$ is the incident angle. As mentioned above, the longitudinal wave function $\Phi(x)=\left[\phi_{A}, \phi_{B}\right]^{T}$ has two components $\phi_{A}$ and $\phi_{B}$, which are corresponding to the wave function of sublattice $A$ and $B$, respectively. Thus the Eq. (1) reduces to the follows:

$$
\left(\begin{array}{cc}
U(x)-E & -i \hbar v_{F}\left(\frac{\partial}{\partial x}+k_{y}\right) \\
-i \hbar v_{F}\left(\frac{\partial}{\partial x}-k_{y}\right) & U(x)-E
\end{array}\right)\left(\begin{array}{l}
\phi_{A} \\
\phi_{B}
\end{array}\right)=0 .
$$

Only $U(x)$ with very simple forms can be solved analytically, for example, the constant potentials in Refs. 2 and 25. In our work, we use the transfer-matrix method, an effective method to solve Eq. (2) with smooth potential $U(x)$ numerically. $^{21,26,27}$ In this method, the central region (nonconstant potential) of graphene can be divided into transversal strips, which are thin enough that the potential in each strip can be viewed as constant. The wave function in the $j$ th strip can be connected with that in the $(j+1)$ th one by the continuing requirement of the wave function, thus the transfer matrix from the $j$ th strip to the $(j+1)$ th strip can be obtained. The overall $T$-matrix is found easily by multiplying all the partial from the left terminal with potential $U_{L}$ to the right end with a constant potential $U_{R}$.

For an explicit example, in the $j$ th strip, the potential $U(x)$ is treated as constant $U_{j}$ as illustrated above. And the solution of Eq. (2) can be expressed as

$$
\begin{aligned}
& \phi_{A}(x)=A_{j} e^{i k_{j} x}+B_{j} e^{-i k_{j} x}, \\
& \phi_{B}(x)=\frac{\hbar v_{F}}{E-U_{j}}\left[\left(k_{j}+i k_{y}\right) A_{j} e^{i k_{j} x}-\left(k_{j}-i k_{y}\right) B_{j} e^{-i k_{j} x}\right],
\end{aligned}
$$

where $k_{j}=\sqrt{\left[\left(E-U_{j}\right) /\left(\hbar v_{F}\right)\right]^{2}-k_{y}^{2}}$. We can rewrite Eq. (3) in matrix form

$$
\left[\begin{array}{l}
\phi_{A}(x) \\
\phi_{B}(x)
\end{array}\right]=M_{j} R_{j}(x) C_{j}
$$

where the matrices $M_{j}, R_{j}$, and $C_{j} \underset{j}{\text { are defined as } M_{j}}$

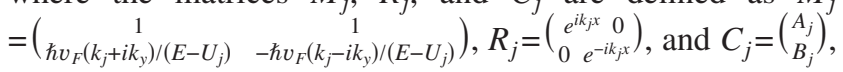
respectively. Using the condition of continuity of wave function at the interface $x=x_{j+1}$ between the $j$ th and the $(j+1)$ th strip, a compact form relation can be found as $M_{j} R_{j}\left(x_{j+1}\right) C_{j}=M_{j+1} R_{j+1}\left(x_{j+1}\right) C_{j+1}, \quad$ therefore $C_{j+1}$ $=R_{j+1}^{-1}\left(x_{j+1}\right) M_{j+1}^{-1} M_{j} R_{j}\left(x_{j+1}\right) C_{j}$. Hence, we define the $T$-matrix between two adjacent strips as follows: $T(j, j+1)$ $=R_{j+1}^{-1}\left(x_{j+1}\right) M_{j+1}^{-1} M_{j} R_{j}\left(x_{j+1}\right)$. Furthermore, the overall $T$-matrix can be obtained straightforwardly by multiplying the series of $T$-matrix in every strip: $T \equiv T(J, J-1) T(J-1, J$ $-2) \ldots T(1,0)$.

In graphene, the holes (below the Dirac points) can contribute to the transmission probability at the Fermi level as

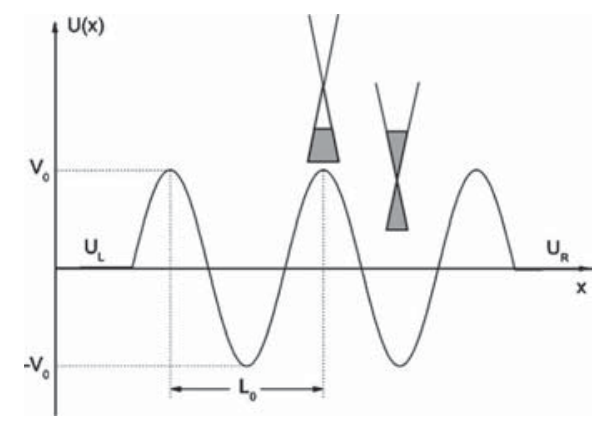

FIG. 1. Schematic diagram of the potential profile in graphene multiquantum well system with two wells.

well as the electrons (above the Dirac points) do. Including the contribution of both charge carriers, the transmission probability through the central part can be derived as ${ }^{26}$

$$
\mathcal{T}=f(s, \alpha)\left\{\begin{array}{l}
1-\left|\frac{T_{21}}{T_{22}}\right|^{2} \quad \text { if }\left(E-U_{L}\right)\left(E-U_{R}\right)>0 \\
1-\left|\frac{T_{22}}{T_{21}}\right|^{2} \text { otherwise. }
\end{array}\right.
$$

Here the angle $\alpha$ is defined as $\cos \alpha=k_{x} / k \equiv \hbar v_{F} k_{x} /\left|E-U_{L}\right|$, and $f(s, \alpha)=f_{1}(\alpha)(s+1) / 2+f_{2}(\alpha)(-s+1) / 2$, where $f_{1}(\alpha)$ $=\Theta(\pi / 2-\alpha)-\Theta(-\pi / 2-\alpha), \quad f_{2}(\alpha)=\Theta(-\pi / 2-\alpha)-\Theta(\pi / 2$ $-\alpha)-\Theta(-\pi-\alpha)+\Theta(\pi-\alpha), s=1$ if $\left(E-U_{L}\right)>0$ and -1 if $\left(E-U_{L}\right)<0$, and $\Theta$ is the standard step function. For details of this procedure, we refer to Nguyen's work in Ref. 26.

The zero-temperature linear conductance ${ }^{26,29}$ can be obtained as

$$
G=\frac{2 g e^{2} W}{v_{F} h^{2}}\left|E_{F}-U_{L}\right| \int_{-\pi}^{\pi} \mathrm{d} \alpha \operatorname{sgn}\left(E_{F}-U_{L}\right) \mathcal{T}\left(E_{F}, \alpha\right) \cos \alpha,
$$

where $e$ is the elementary charge, $g=4$ is the degeneracy of electron states, $W$ is the sample width, and $E_{F}$ is the equilibrium Fermi energy. The expression for conductance is rather general and can be used for any smooth potential.

\section{RESULTS AND DISCUSSION}

In order to investigate the influence of the multiquantum well potential on the transport properties, we consider the potential $U(x)$ as follows:

$$
U(x)=\left\{\begin{array}{ll}
U_{L}=0 & x<0 \\
V_{0} \sin \left(G_{0} x\right) & 0<x<L \\
U_{R}=0 & x>L
\end{array},\right.
$$

where $V_{0}$ is the amplitude of the sine potential, $L=(N$ $+1 / 2) L_{0}$ is the length of central region, $L_{0}$ is the width of a quantum well, and $G_{0}$ is the inverse of the width $L_{0}: G_{0}$ $=2 \pi / L_{0}$. The potential profile can be seen in Fig. 1 . The amplitude of the oscillating potential $V_{0}$ is assumed to be 50 $\mathrm{meV}$, setting the zero-potential point at $U_{L}$ (or $U_{R}$ ). The same procedure is adopted about the relation of the incident angle $\beta$ and $\alpha$ in Eq. (5) as Ref. 26. Hereafter, we will address the influence of the quantum well number and the quantum well 


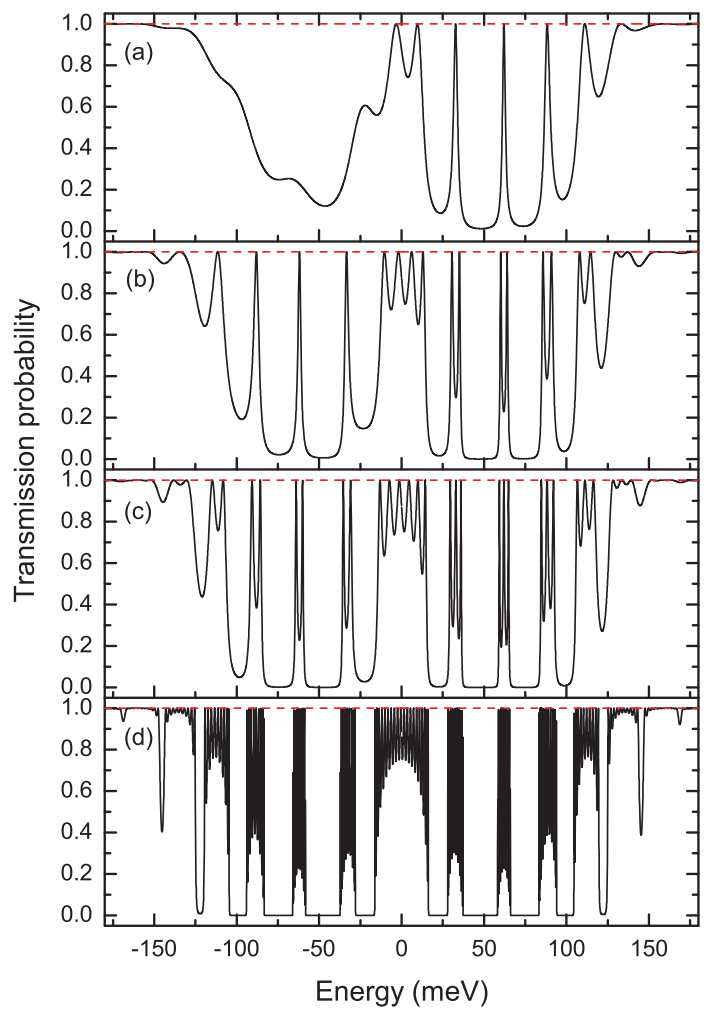

FIG. 2. (Color online) The transmission probability $\mathcal{T}$ as a function of incident energy with the same incident angle for different well number (a) $N$ $=1$, (b) $N=2$, (c) $N=3$, and (d) $N=10$. The solid lines and the dashed lines correspond to incident angle $\beta=\pi / 6$ and $\beta=0$, respectively. The width of a quantum well is $L_{0}=100 \mathrm{~nm}$.

width on the anisotropic transmission probability and the conductance.

To understand the impact of the quantum well number on the transport property, we calculated the transmission probability spectra with different well number. The numerical results are presented in Fig. 2. We found that the central part is completely transparent at normal incidence $(\beta=0)$ in despite of the quantum well number, seen from the dashed curves in Fig. 2. Owing to the conservation of pseudospins ${ }^{4}$ in graphene, back-scattering process is suppressed at normal incidence, which makes the central region transparent. A transmission gap appears for tilted incidence, which is associated with evanescent wave in the barrier. ${ }^{25,26}$ The quantum well for electrons is a quantum barrier for holes in graphene in virtue of its chiral nature. ${ }^{2}$ Thus, when the quantum well number is one, in the negative energy range, there is another transmission gap emerging, because the charge carriers in this range are holes, and the quantum well for electrons now becomes a barrier, shown by the solid line in Fig. 2(a). The most impressive feature is the appearance of a few acute peaks in the transmission gap of the positive energy range, which are defined as transmission windows here. When the incident energy is aligned with the bound states in the quantum well, the transmission is greatly enhanced by resonant tunneling. The central region has a equivalent quantum well profile for holes when the quantum well number is two, as shown in Fig. 1. Therefore, resonant peaks also emerge in the negative transmission gap, and the fine structure of the posi-

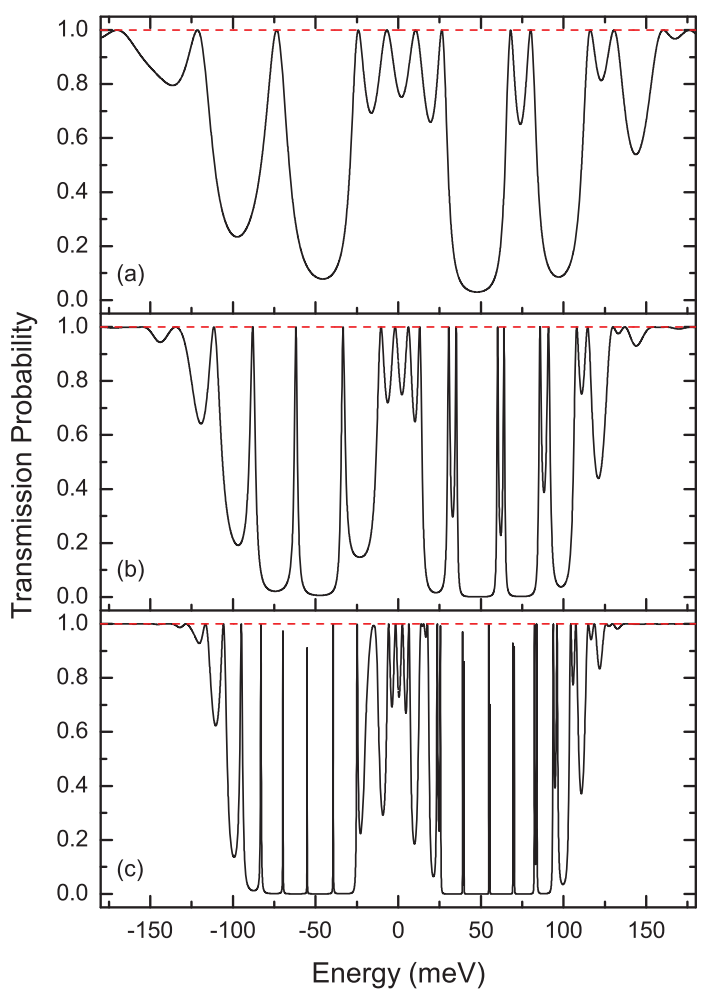

FIG. 3. (Color online) The transmission probability $\mathcal{T}$ as a function of incident energy $E$ with the same incident angle for different quantum well width (a) $L_{0}=50 \mathrm{~nm}$, (b) $L_{0}=100 \mathrm{~nm}$, and (c) $L_{0}=200 \mathrm{~nm}$. The solid lines and the dashed lines correspond to incident angle $\beta=\pi / 6$ and $\beta=0$, respectively. The number of quantum wells is 2 .

tive transmission windows in Fig. 2(a) becomes two, as illustrated in Fig. 2(b). The bound states in the adjacent quantum wells couple to each other to form the transmission windows with two peaks. The similar resonant tunneling related to bound states has been stated in semiconductor. ${ }^{29}$ When the quantum well number increases to three [the curves in Fig. 2(c)], the fine structure of the transmission windows is three in the positive transmission gap, while the fine structure of that in negative transmission gap is two because the quantum well number for holes is two. The transmission windows develop into minibands when the number of quantum wells is very large, as shown in Fig. 2(d). The location and the width of the transmission windows are independent of the quantum well number, as can be seen in Fig. 2. And the quantum well number determines the fine structure of the transmission windows. The transmission windows in transmission gaps have a potential application, which admit the transmission of the electrons with special energies. Compared with the results in Ref. 23, Fig. 2(d) has the similar features, the transmission gap and the transmission windows with resonant peaks. However, owing to the sine potential in our work, the quantum wells for electrons become quantum barriers for hole, thus the transmission gap and the transmission windows emerge in the negative energy range, which are different with the case in Ref. 23.

Since the energy of bound states are insensitive to the quantum well number, we have calculated the transmission probability with fixed quantum well number $N=2$ for varying quantum well width in Fig. 3. The central region is com- 


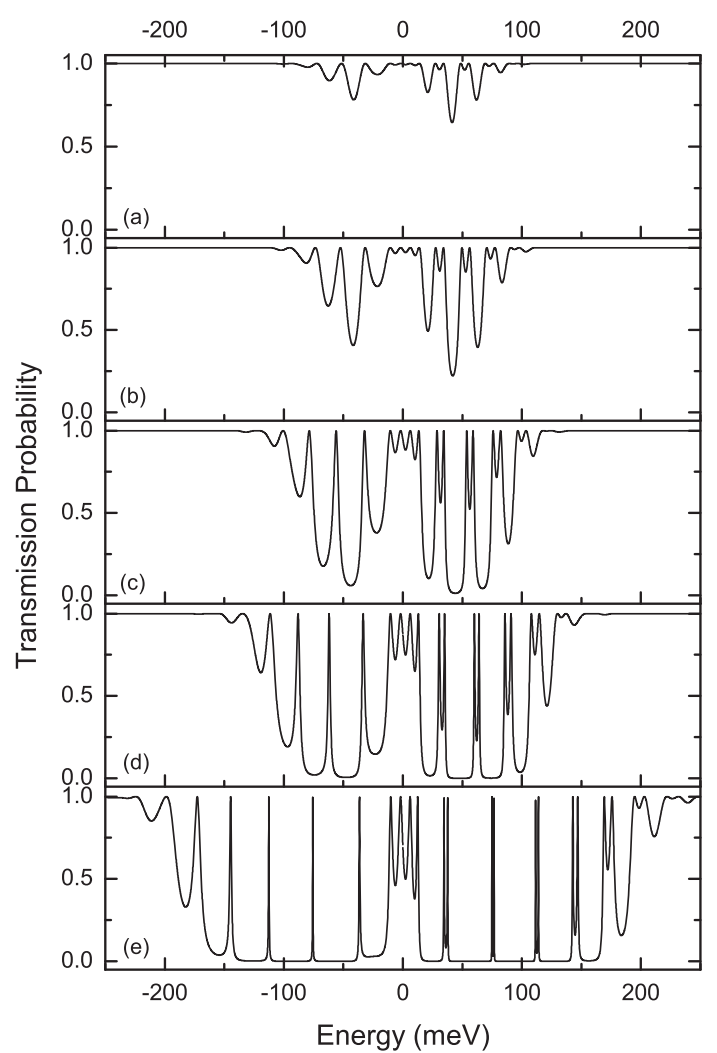

FIG. 4. The transmission probability $\mathcal{T}$ as a function of incident energy with the same quantum well number $N=2$ for different incident angle: (a) $\beta$ $=\pi / 36$, (b) $\beta=\pi / 18$, (c) $\beta=\pi / 9$, (d) $\beta=\pi / 6$, and (e) $\beta=\pi / 4$. The quantum well width is $100 \mathrm{~nm}$.

pletely transparent for normal incidence $(\beta=0)$, no matter how large the quantum well width is, as indicated from the dash lines in Fig. 3. Increasing the quantum well width, the transmission probability decays in the form of $e^{-\kappa d}$ within the transmission gaps range. The resonant peaks in one transmission window are much sharper and closer, which infers that the interference plays a significant role in the transport for a larger width. The energy difference between two adjacent transmission windows decrease with increasing the quantum well width, which indicates that the bound states are denser for a larger quantum well width. The changing of the width shifts the bound states between two adjacent quantum wells, so the energy location and the width of the transmission windows are varying correspondingly. The number of the resonant peaks in one positive transmission window is two and that in one negative transmission window is one due to the quantum well number $N=2$. Thus, the quantum well width determines the energy location and the width of the transmission windows, and the quantum well number only gives the fine structure of the transmission windows.

Another factor to influence the transmission probability is the incident angle. A set of the transmission probability for varying incident angle is shown in Fig. 4. There is no transmission gap at the normal incidence, which is illustrated in Figs. 2 and 3. As the incident angle increases, the evanescent wave appears in the barrier part, thus the transmission gaps and the transmission windows emerge in the positive and negative energy range in Fig. 4(a). Owing to the relation $k_{j}$

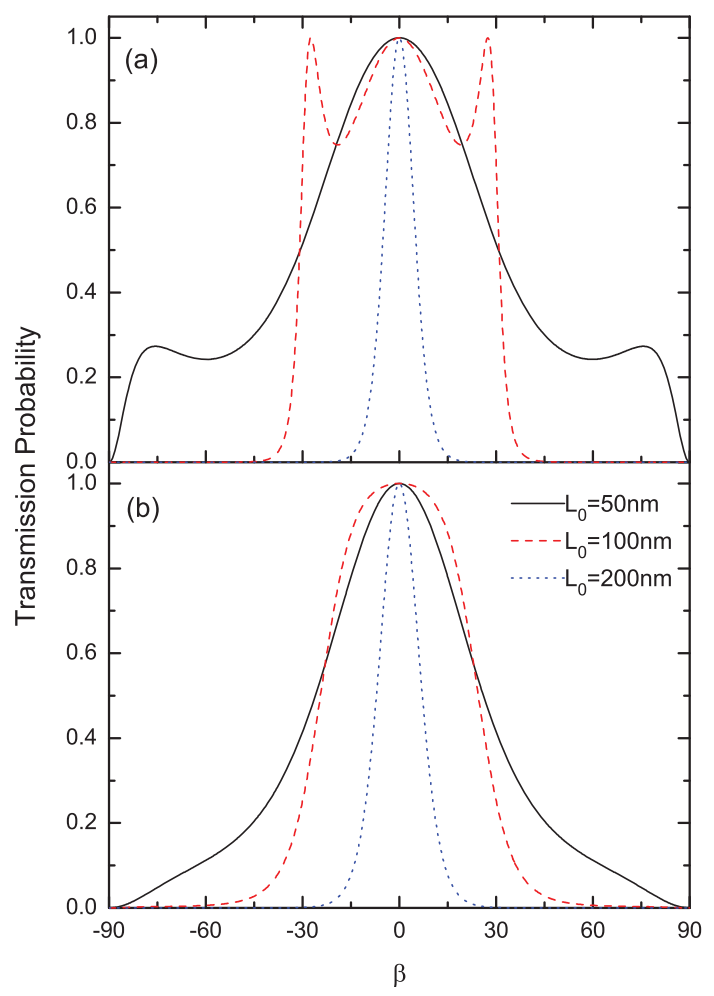

FIG. 5. (Color online) The transmission probability $\mathcal{T}$ as a function of incident angle $\beta$ with the same well number $N=2$ for different incident energy (a) $E=30 \mathrm{meV}$ and (b) $E=-30 \mathrm{meV}$. The solid line, the dashed line, and the dotted line correspond to $L_{0}=50 \mathrm{~nm}, L_{0}=100 \mathrm{~nm}$, and $L_{0}=200 \mathrm{~nm}$, respectively.

$=\sqrt{\left[\left(E-U_{j}\right) /\left(\hbar v_{F}\right)\right]^{2}-\left[\left(E-U_{L}\right) /\left(\hbar v_{F}\right) \sin \beta\right]^{2}}$, the contribution to the propagating wave number decreases for a large incident angle. Therefore, the transmission gaps become wider increasing incident angle, which is evident from Figs. 4(a)-4(e). For the same reason, the locations of the transmission windows induced by the multiquantum well structure have also changed, and the widths of the transmission windows become narrower. The incident angle affects drastically the properties of transmission gaps and the transmission windows, which indirectly testifies the anisotropic characteristics in a graphene multiquantum well system.

In real devices, the electron transport is drastically different from the ballistic regime we assumed due to the existence of disorder in graphene. The defects in graphene strongly modify the states near Dirac point, ${ }^{5,7}$ for example, ripples are crucial for the production of stable graphene, ${ }^{30}$ vacancies change the density of states close to the Fermi level, ${ }^{31}$ the charged impurities induce a particle-hole asymmetry, ${ }^{32}$ and so on. The inserting of defects would change the density of states, leading to the enhancement or suppression of coherent electron transport. The effect of disorder is the subject of our next work.

The transmission probability as a function of the incident angle for different quantum well width with fixed incident energy is presented in Fig. 5. Obviously, the potential is always perfectly transparent for incident angles approaching to the normal incidence $\beta=0$ due to the Klein tunneling, regardless of the quantum well number and the quantum well width. The transversal wavevector is enhanced with increas- 


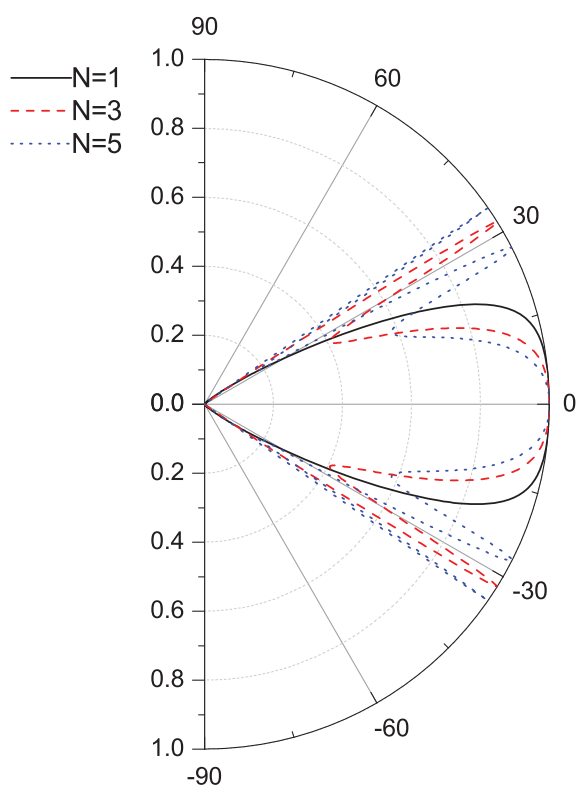

FIG. 6. (Color online) The transmission probability $\mathcal{T}$ as a function of incident angle $\beta$ with the incident energy $E=30 \mathrm{meV}$ for different quantum well number. The solid line, the dashed line, and the dotted line correspond to $N=1, N=3$, and $N=5$, respectively. The width of a quantum well is $L_{0}$ $=100 \mathrm{~nm}$.

ing the incident angle, which in turn leads to the reduction in the transmission probability. The incident energy is assumed to be $30 \mathrm{meV}$ in Fig. 5(a), more than $U_{L}$, which indicates the transparency is mostly associated with the transport of electrons through an upward potential. While the incident energy is assumed to be $-30 \mathrm{meV}$ in Fig. 5(b), less than $U_{L}$, which means the transport is mainly associated with holes through a downward potential. The transmission probability is suppressed by the interference effect with increasing the quantum well width in Fig. 5. This feature is easily understood in common sense that an electron can go through a short potential more easily than through a long one. Increasing the quantum well width not only reduces the amplitude of the transmission probability, but also augments the zero- $\mathcal{T}$ incident angle range due to the interference effect in multiquantum well potential, as seen in Fig. 5. Given a fixed well width $L_{0}=100 \mathrm{~nm}$ and the incident energy $E=30 \mathrm{meV}$, the dependence of transmission probability on the quantum well number is presented in Fig. 6. The increasing of well number makes the anisotropic characteristics more obvious. The reason is that the interference plays an important role in the transport process, which is similar with the results in Ref. 21.

The effective conductance $G^{*}=G / W$ (the conductance divided by the sample width) as a function of Fermi energy, calculated from Eq. (6), is given in Fig. 7 for the same structures as those discussed in Fig. 6. The effective conductance $G^{*}$ vanishes at Fermi energy $E_{F}=0$ in despite of the quantum well number due to the contribution of the term $\left|E_{F}-U_{L}\right|$ in Eq. (6) (here $U_{L}=0$ ). In the low negative and high positive energy range, the dependence of transmission on the incident angle is almost the same with each other, so the conductance becomes linear with the difference $\left|E_{F}-U_{L}\right|$. But the multiquantum well structure brings a few unique features. One consequence is that the conductance curves oscillate with

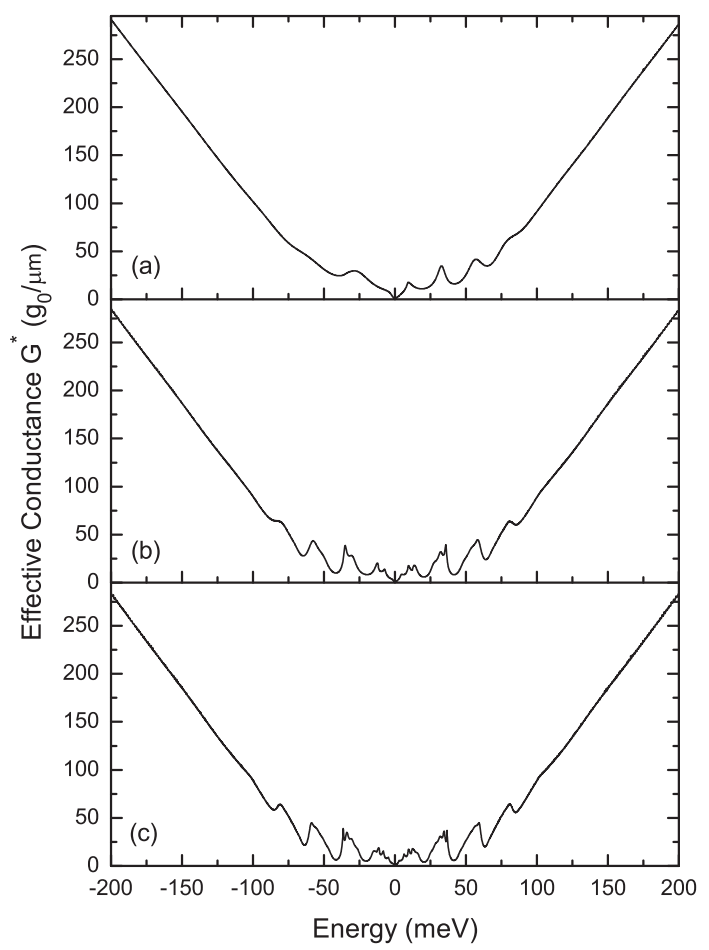

FIG. 7. (Color online) The effective conductance $G^{*}$ as a function of incident energy for different well number (a) $N=1$, (b) $N=3$, and (c) $N=5$. The quantum well width $L_{0}$ is $100 \mathrm{~nm}$. The units of $G$ and $W$ are taken as $g_{0}$ $=2 e^{2} / h$ and micrometer, respectively.

very small amplitudes in the low negative and the high positive energy range in Figs. 7(b) and 7(c). This new characteristics should be related with the transmission windows corresponding to bound states induced by the multiquantum well potential. The absence of this tiny oscillation in the low negative energy range and the emergence in the high positive energy range in Fig. 7(a) prove the above explanation. The obvious oscillation in the middle energy range $\left(-V_{0}, V_{0}\right)$ is attributed to the anisotropic characteristics of charge carriers through a single quantum well, as shown in Fig. 7(a). The curve in Fig. 7(b) and is similar with the curve in Fig. 7(c) except for some details in virtue of the different quantum well number. The relative low value of conductance in the middle energy range is partly due to the small value $\mid E_{F}$ $-U_{L} \mid$ and partly due to the anisotropic property in graphene. The effect of the quantum well width on the conductance for quantum well number $N=2$ is presented in Fig. 8. As the discussion about Fig. 3, the variability of quantum well width changes the features of the transmission windows induced by the multiquantum well potential. The increasing of the quantum well width makes the oscillation more drastic in the middle energy range owing to the increasing of the resonant states. All these oscillating features may be helpful in explaining the oscillatory characteristics in experiment. ${ }^{9}$

\section{CONCLUSION}

We have studied the transport properties of a graphene multiquantum wells system using the transfer-matrix method. The feature of perfect transmission is testified at normal incidence regardless of the quantum well number and 


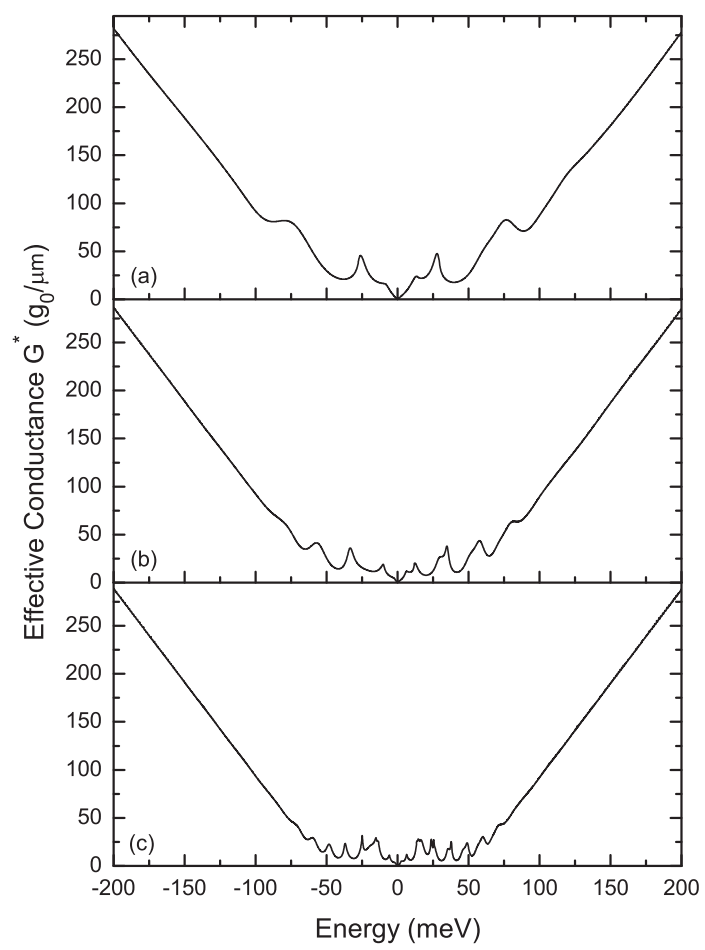

FIG. 8. The effective conductance $G^{*}$ as a function of incident energy for different quantum well width (a) $L_{0}=50 \mathrm{~nm}$, (b) $L_{0}=100 \mathrm{~nm}$, and (c) $L_{0}$ $=200 \mathrm{~nm}$. The quantum well number is 2 . The units are the same as that in Fig. 6.

the quantum well width. For tilted incidence, there are two types of transmission gaps in the transmission spectra through a multiquantum well system. One emerges in the positive energy range for electrons and the other one emerges in the negative energy range for holes. Besides, a few transmission windows appear in both transmission gaps. The formation of a transmission window is related to the bound states in the quantum wells. The bound states in adjacent quantum wells couple to each other, and form a transmission window. The number of the quantum wells gives the fine structure of the transmission windows. For example, the system with $N$ quantum wells determines that the number of peaks in one positive transmission window is $N$ and the number of peaks in one negative transmission window is $(N$ $-1)$. The location and the width of the transmission windows are sensitive to the quantum well width and the incident angle, and the quantum well number only determines the number of resonant peaks in one transmission window. We also have demonstrated the anisotropic features for different quantum well number and quantum well width. The increase in quantum well width makes the nonzero-transmission angle range decreased. The influence of the quantum well number is the enhancement of the interference effect. Some novel features appear in the conductance curves of the multiquantum well systems, such as, tiny oscillation in the low negative and the high positive energy range, the fine structures in the middle energy range. The main effect of the quantum well width is the obvious conductance oscillation in the middle energy range. These properties might be useful to understand the experimental conductance oscillation. ${ }^{9}$

\section{ACKNOWLEDGMENTS}

This work was supported by the National Basic Research Program of China (Project No. 2007CB310402), the National Natural Science Foundation of China (Project No. 60721004), the major projects (Project Nos. KGCX1-YW-24 and KGCX2-YW-231) of the Chinese Academy of Sciences (CAS), and "Hundred Scholar Plan" of CAS.

${ }^{1}$ K. S. Novoselov, A. K. Geim, S. V. Mrozov, D. Jiang, Y. Zhang, S. V. Dubonos, I. V. Grigorieva, and A. A. Firsov, Science 306, 666 (2004).

${ }^{2}$ M. I. Katsnelson, K. S. Novoselov, and A. K. Geim, Nat. Phys. 2, 620 (2006).

${ }^{3}$ Y. Zhang, Y. W. Tan, H. L. Stormer, and P. Kim, Nature (London) 438 , 201 (2005).

${ }^{4}$ C. W. J. Beenakker, Rev. Mod. Phys. 80, 1337 (2008).

${ }^{5}$ A. H. Castro Neto, F. Guinea, N. M. R. Peres, K. S. Novoselov, and A. K. Geim, Rev. Mod. Phys. 81, 109 (2009)

${ }^{6}$ O. Klein, Z. Phys. 53, 157 (1929).

${ }^{7}$ N. M. R. Peres, J. Phys.: Condens. Matter 21, 323201 (2009).

${ }^{8}$ N. Stander, B. Huard, and D. Goldhaber-Gordon, Phys. Rev. Lett. 102, 026807 (2009).

${ }^{9}$ A. F. Young and P. Kim, Nat. Phys. 5, 222 (2009).

${ }^{10}$ V. P. Gusynin and S. G. Sharapov, Phys. Rev. Lett. 95, 146801 (2005).

${ }^{11}$ E. McCann and V. I. Falko, Phys. Rev. Lett. 96, 086805 (2006).

${ }^{12}$ K. S. Novoselov, E. McCann, S. V. Morozov, V. I. Falko, M. I. Katsnelson, U. Zeitler, D. Jiang, F. Schedin, and A. K. Geim, Nat. Phys. 2, 177 (2006).

${ }^{13}$ C. Bai, Y. Yang, and X. Zhang, Appl. Phys. Lett. 92, 102513 (2008).

${ }^{14}$ C. W. J. Beenakker, Phys. Rev. Lett. 97, 067007 (2006).

${ }^{15}$ J. C. Cuevas and A. L. Yeyati, Phys. Rev. B 74, 180501(R) (2006).

${ }^{16}$ B. Huard, J. A. Sulpizio, N. Stander, K. Todd, B. Yang, and D. GoldhaberGordon, Phys. Rev. Lett. 98, 236803 (2007).

${ }^{17}$ J. R. Williams, L. DiCarlo, and C. M. Marcus, Science 317, 638 (2007).

${ }^{18}$ J. M. Pereira, Jr., P. Vasilopoulos, and F. M. Peeters, Appl. Phys. Lett. 90, 132122 (2007).

${ }^{19}$ J. M. Pereira, Jr., V. Mlinar, F. M. Peeters, and P. Vasilopoulos, Phys. Rev. B 74, 045424 (2006).

${ }^{20}$ N. Abedpour, A. Esmailpour, R. Asgari, and M. R. R. Tabar, Phys. Rev. B 79, 165412 (2009).

${ }^{21}$ C. Bai and X. Zhang, Phys. Rev. B 76, 075430 (2007).

${ }^{22}$ L. Brey and H. A. Fertig, Phys. Rev. Lett. 103, 046809 (2009).

${ }^{23}$ M. Barbier, F. M. Peeters, P. Vasilopoulos, and J. M. Pereira, Jr., Phys. Rev. B 77, 115446 (2008).

${ }^{24}$ Y. P. Bliokh, V. Freilikher, S. Savel'ev, and F. Nori, Phys. Rev. B 79, 075123 (2009).

${ }^{25}$ X. Chen and J. Tao, Appl. Phys. Lett. 94, 262102 (2009).

${ }^{26}$ H. C. Nguyen and V. L. Nguyen, J. Phys.: Condens. Matter 21, 045305 (2009).

${ }^{27}$ C.-H. Park, L. Yang, Y.-W. Son, M. L. Cohen, and S. G. Louie, Nat. Phys. 4, 213 (2008).

${ }^{28}$ L. Brey and H. A. Fertig, Phys. Rev. B 73, 235411 (2006).

${ }^{29}$ S. Datta, Electronic Transport in Mesoscopic Systems (Cambridge University Press, Cambridge, 1995).

${ }^{30}$ J. C. Meyer, A. K. Geim, M. I. Katsnelson, K. S. Novoselov, T. J. Booth, and S. Roth, Nature (London) 446, 60 (2007).

${ }^{31}$ N. M. R. Peres, F. Guinea, and A. H. Castro Neto, Phys. Rev. B 73, 125411 (2006).

${ }^{32}$ X. Du, I. Skachko, A. Barker, and E. Y. Andrei, Nat. Nanotechnol. 3, 491 (2008). 